\title{
Current analytical methods for porcine identification in meat and meat products
}

\begin{abstract}
Authentication of meat products is critical in the food industry. Meat adulteration may lead to religious apprehensions, financial gain and food-toxicities such as meat allergies. Thus, empirical validation of the quality and constituents of meat is paramount. Various analytical methods often based on protein or DNA measurements are utilized to identify meat species. Protein-based methods, including electrophoretic and immunological techniques, are at times unsuitable for discriminating closely related species. Most of these methods have been replaced by more accurate and sensitive detection methods, such as DNA-based techniques. Emerging technologies like DNA barcoding and mass spectrometry are still in their infancy when it comes to their utilization in meat detection. Gold nanobiosensors have shown some promise in this regard. However, its applicability in small scale industries is distant. This article comprehensively reviews the recent developments in the field of analytical methods used for porcine

identification.
\end{abstract}

Keyword: Meat adulteration; ELISA; Species specific PCR; Nanobiosensor; DNA barcoding; Mass spectrometry; Digital PCR' Loop-mediated isothermal amplification (LAMP); Electronic noses; Real time PCR 